\title{
Human proteome: New research technologies for cancer research
}

\author{
More M.K. ${ }^{1}$, Nair V.K. ${ }^{2}$, Thikekar V.P. ${ }^{3}$, Sawant J.J. ${ }^{4}$ Ravidhone Y. ${ }^{5}$, Patil A. ${ }^{6}$, Paul Andrea. ${ }^{7}$ \\ ${ }^{1}$ Tilak College of Science and Commerce, Vashi, Navi Mumbai, India \\ ${ }^{2}$ Bhavan's College, Andheri (W), Mumbai- 400058, India \\ ${ }^{3}$ Bhartiya Gramin Punarrachana Sanstha's Arts, Commerce and Science, Wadala,Mumbai, India \\ ${ }^{4}$ Gogate Jogalekar College of Science, Arts and Commerce, Ratnagiri, India \\ ${ }^{5}$ Elphinstone College, Mumbai, India \\ ${ }^{6}$ V.E.S., College of Arts, Science and Commerce, Chembur, Mumbai-400 071, India \\ ${ }^{7}$ Sophia College, Grand road, Mumbai, India
}

\begin{abstract}
Proteins are vital parts of living organisms, as they are the main components of the physiological metabolic pathways of cells. Protein profiling is recognized \& powerful method of classifying new protein families and to use conserved region within multiple alignment of the related protein. The human proteome is dynamic, changing constantly in response to the needs of the body. The proteome also changes in response to cancer and other diseases, making the proteome of great interest to medical researchers. Cancer research ranges from epidemiology, molecular bioscience (bench research) to the performance of clinical trials to evaluate and compare applications of the various cancer treatments. These applications include surgery, radiation therapy, chemotherapy and hormone therapy.
\end{abstract}

Keywords- Proteome, Cancer, Protein profiling, Interactome, Systems biology, Omics network

Protein profiling and interactome in proteomics The term proteome refers to the totality of the proteins in a cell, tissue, or organism. Proteomics is the study of these proteins, their identity, their biochemical properties and functional roles, and how their quantities, modifications, and structures change during development and in response to internal and external stimuli. It is the quantitative assessment of the protein expression level. The present study examined to what extent the cellular perturbations involve changes in gene expression [51]. Plasma protein profiling using separations coupled to matrix-assisted laser desorption ionization mass spectrometry (MALDI MS) has great potential in translational research as it can be used for biomarker discovery and contribute to disease diagnosis and therapy Multiple profiling and identification experiments can be conducted on the same protein fractions because only $5 \%$ of the fractions are used for MALDI MS profiling [1]. The ongoing development of high-throughput proteomic profiling technologies will facilitate the identification of molecular signatures that are associated with bladder disease. . The appropriate use of these approaches has the potential to provide efficient biomarkers for the early detection and monitoring of recurrent bladder cancer [2]. Recent studies focuses on the accumulating proteomic signatures of breast tumor progression, particularly those that correlate with the occurrence of distant metastases, and discuss some of the expected future developments in the field [3]. The comparion of protein data to a previously published microarray dataset shows that mRNA and protein expression are poorly correlating. One of the first examples of a large-scale quantitative proteomics experiment in Caenorhabditis elegans shows the potential and feasibility of an approach enabling system-wide accurate quantitative proteomics experiments in this model organism [52]. Interactome is terms of predicted permanent protein complexes and predicted transient, nongeneric interactions between these complexes. The interactome is generated by means of an associated computational algorithm, from raw highthroughput affinity purification/mass spectrometric interaction data [4]. Tax interactome will have significant utility toward defining new and understanding known activities of this important viral protein.Current research constructed an in silico Tax interactome comprised of 46 literature-confirmed proteinprotein interactions. This number was then reduced to four Tax-interactions suspected to play a role in DNA damage response (Rad51, TOP1, Chk2, 53BP1). The first-neighbor and second-neighbor interactions of these four proteins were assembled from available human protein interaction databases [5]. The merging of a viral interactome and the human interactome has been used to simulate viral infection, paving the way for future studies aiming at providing molecular basis of human diseases [6]. Recent findings identify a methylation-directed protein interaction mechanism in germ cells mediated by germline Tudor domains and methylated Piwi family proteins, and suggest a complex mode of regulating the organization and function of Piwi proteins in piRNA silencing pathways [49]. We can summarize the molecular network of Grb2 in $B$ cells and introduce a proteomic approach to elucidate the interactome of Grb2 in vivo [50]. For example, cancer cells often secrete specific proteins or fragments of proteins into the bloodstream and other bodily fluids, such as urine and saliva. Cancer is a kind of disease in which cells divide abnormally without control and may overrun other tissues. Through blood and other lymph system these cells spread in all over body. Cancer results in death if spread of cells is 
not controlled. Cancer-related fatigue can be measured as both a unidimensional and a multidimensional construct. Unidimensional fatigue and its symptom correlates have undergone some previous investigation; however, minimal research has considered the differential effect of correlates on individual dimensions of fatigue.

\section{Systems Biology and Omics Network}

Systems biology is a biology-based interdisciplinary study field that focuses on the systematic study of complex interactions in biological systems.Systems biology is to discover new emergent properties that may arise from the systemic view used by this discipline in order to understand better the entirety of processes that happen in a biological system [7]. A mathematical framework is used to simulate the effects on the growth of a pathogen when enzymes in its metabolic pathways are inhibited. Here study developed a mathematical framework in which enzyme inhibition kinetics, metabolic network simulation, and cell growth dynamics are considered together to produce a system that is able to quantitatively model drug inhibition of cell growth. Recent study separately simulated the effects of two metabolic inhibitors, 3-NP and sAMS, on the growth of $M$. tuberculosis cells, using an in vitro media model designed to mimic the limited nutritional environment in host cell [8]. An integrated investigation of network and highthroughput information together can improve the quality of the analysis by accounting simultaneously for topological network properties alongside intrinsic features of the high-throughput data [9]. The network is predicted by the Pathway Tools software using MetaCyc as a reference, which consists of metabolites, enzymes, reactions and metabolic pathways. Omics is that enable visualization of omics datasets on two different genome-scale diagrams and tools for comparative analysis [53]. Reverse engineering of high-throughput omics data to infer underlying biological networks is one of the challenges in systems biology. However, applications in the field of metabolomics are rather limited. Studies are focused on a systematic analysis of metabolic network inference from in silico metabolome data based on statistical similarity measures [10]. The availability of pathogen and host omics data in an integrated framework allows global analysis of the data and comparisons across different experiments and organisms [56]. The Soybean Proteome Database aims to be a data repository for functional analyses of soybean responses to flooding injury, recognized as a major constraint for establishment and production of this plant [55]. Analysis of microarray, SNPs, proteomics, and other high-throughput (OMICs) data is challenging because of its biological complexity and high level of technical and biological noise. One way to deal with both problems is to perform analysis with a high-fidelity annotated knowledge base of protein interactions, pathways, and functional ontologies [11]. The archaea, the thermoacidophilic crenarchaeote Sulfolobus solfataricus has become an important model organism for physiology and biochemistry, comparative and functional genomics, as well as, more recently also for systems biology approaches [54].

\section{Clinical proteomics}

Clinical Proteomics provides a scholarly forum for novel scientific research in the field of translational proteomics. Mast cells are widely recognized as effector cells of allergic inflammatory reactions [57]. Proteomics of brain, neck and tumor cancer; in neuroscience, a host of applications was proposed ranging from neurotoxicology, neurometabolism, determination of the proteome of the individual brain areas in health and disease. The principle consists of twodimensional gel electrophoresis with in-gel protein digestion of protein spots and identification by mass-spectrometrical approaches [12]. The TMZ-BioShuttle dramatically enhanced the potential of $T M Z$ for the treatment of brain tumors and is an attractive drug for combination chemotherapy [48]. Head and neck squamous cell carcinomas (HNSCCs) for differences in protein expression between oral cavity, oropharynx, larynx, and hypopharynx subsites. Retrospective proteomic analysis using tissue microarray (TMA) and 2-dimensional difference gel electrophoresis (2D-DIGE); automated quantitative protein expression analysis was used to interrogate levels of 4 cellcycle regulatory proteins chosen for their known roles in cancer [13]. For a better understanding of the molecular mechanisms behind the process of tumorigenesis and tumor progression, studies have analyzed changes of protein expression between microdissected normal pharyngeal epithelium and tumor tissue by Protein Chip technology. Biomarker in head and neck cancer can be found, identified, and assessed by combination of Protein Chip technology, twodimensional gel electrophoresis, and immunohistochemistry [14]. The results are validated by Western blotting analysis and results showed that enolase-alpha, annexin-I and annexin-II might be important molecules in head and neck cancer invasion and metastasis. It is an important complementary role for proteomics in identification of molecular abnormalities important in cancer development and progression [15].

\section{Proteomics of breast cancer}

Estrogen, hormones and growth factors in serum play important roles in the carcinogenesis and development of breast cancer. The effect of the 
above factors on protein components and their contents in breast cancer cells is necessary for understanding the molecular mechanisms. In this study, isobaric tags for relative and absolute quantification (iTRAQ) labeling coupled with twodimensional liquid chromatography-tandem mass spectrometry (2D-LC-MS/MS) was employed to compare the differential protein expressions in MCF7 cell.[16] Protein glycosylation represents one of the major post-translational modifications and can have significant effects on protein function. Moreover, changes in the carbohydrate structure are increasingly being recognized as an important modification associated with cancer etiology. As a result, several glycoproteins including alpha-1B-glycoprotein, complement C3, alpha-1-antitrypsin and transferrin can be identified [17]. Scientist's goal was to learn what the immune system "sees" on the surfaces of tumor cells by acid-eluting peptides from HLA molecules for extended time periods. Researchers determined how long peptides would continue to elute over time from a pancreatic tumor cell line, Panc-1, and a breast cancer cell line, MCF-7, at pH 3.0 in citrate buffer such that the findings provide sequences and gene names of peptides presented by $\mathrm{MHC}$ class I molecules from common pancreas and breast cancer cell lines [18].

\section{Proteomics of colorectal and colon cancer}

Chronic inflammation is a risk factor for colorectal cancer (CRC) development. The aim of this study is to determine the differences in protein expression between $\mathrm{CRC}$ and the surrounding nontumorous colonic tissues in the mice that received azoxymethane (AOM) and dextran sodium sulfate (DSS) using a proteomic analysis [19]. To identify the oncofetal proteins involved in CRC carcinogenesis, differentially expressed proteins among fetal colorectal tissues, CRC, and the paired tumor-adjacent normal colorectal tissues were investigated by a two-dimensional gel electrophoresis and MALDI-TOF/TOF-based proteomics approach. The desmin should be considered as a potential oncofetal serum tumor marker for $\mathrm{CRC}$ that may have significance in the detection of patients with CRC [20] Also are the discoveries of over expression of GPD1 and RRBP1 proteins and lack of expression for HNRNPH1 and SERPINB6 proteins which are new candidate biomarkers of colon cancer [21].

\section{Proteomics of lung cancer}

In Both lung cancer and chronic obstructive pulmonary disease (COPD) are associated with cigarette smoking, which is generating reactive oxidant species, induces a chronic inflammatory state in the lung. Activation, particularly of nuclear factor- B, occurs in both cancer and COPD, and expression of a number of genes is altered in both diseases. In lung cancer, DNA damage, lack of DNA repair, and genomic instability predominate, whereas matrix degradation, lack of repair, and an intense immune response predominate in COPD. The reasons for the different responses to a common inflammatory response induced by smoking remain to be determined, but likely lie in genetic polymorphisms in genes that regulate genome integrity in cancer and that regulate the immune response to tissue destruction in COPD [22]. The combination of multi-dimensional liquid chromatography and two-dimensional difference gel electrophoresis provides a valuable tool for serum proteomics in lung cancer [23].

\section{Proteomics of esophageal cancer}

Lymph node metastasis is a major prognostic factor for esophageal squamous cell carcinoma (ESCC). In recent years, endoscopic mucosal resection (EMR) has been developed with excellent results for the treatment of the superficial ESCC. To make the EMR treatment successful, it is important to establish a good indicator to identify ESCC patients at a high risk of lymph node metastasis [24]. To develop a prognostic biomarker for esophageal squamous cell carcinoma (ESCC), researchers examined the proteomic profile of ESCC using twodimensional difference gel electrophoresis (2DDIGE), and identified proteins associated with prognosis by mass spectrometry [25]. The microarray analysis identified 24 proteins with aberrant expression in esophageal cancer compared with the corresponding normal mucosa. These proteins play a key role in the NF-kB pathway, and NF-kB is a signal transduction factor that has emerged as an important modulator of altered gene programs and malignant phenotype in the development of cancer. The association of these proteins with esophageal cancer may indicate that mitotic checkpoint gene products and $\mathrm{NF}-\mathrm{KB}$ play an important part [26]. Also the over expression of CD24, which was correlated with Ki-67 expression, is a novel independent prognostic marker for identifying patients with poor prognosis after curative resection of esophageal squamous cell carcinoma [27].

Proteomics of hepatic and pancreatic cancer The liver is an organ with paradoxic immunologic properties and is known for its tolerant microenvironment, which holds important implications for hepatic diseases. Our rereveal is that LSECtin is a novel regulator of $T$ cells and expose a crucial mechanism for hepatic T-cell immune suppression [28]. The differential expression of proteins in cancer were analyzed by imaging analysis and MALDI-TOF-MS after the total protein was extracted from the hepatic fibrosis tissue and the normal liver tissue by 2DE. Some differentially expressed proteins have 
been found in the hepatic fibrosis tissue and the normal liver tissue. Therefore these data provided a fundamental basis for further study of the mechanism of hepatic fibrogenesis [29]. Protein microarrays have been used to explore whether a humoral response to pancreatic cancer-specific tumor antigens has utility as a biomarker of pancreatic cancer. To determine if such arrays can be used to identify novel autoantibodies in the sera from pancreatic cancer patients, proteins from a pancreatic adenocarcinoma cell line (MIAPACA) were resolved by $2-D$ liquid-based separation. This work demonstrates the use of natural protein arrays to study the humoral response as a means to search for the potential markers of cancer in serum.[30] MALDI-TOF MS-based serum peptidome profiling allowed the discovery and validation of platelet factor 4 as a new discriminating marker in pancreatic cancer [31]. The detection of autoantibodies to calreticulin isoforms may have utility for the early diagnosis of pancreatic cancer [32].

\section{Proteomics of leukemia cancer}

Acute leukemia is a heterogeneous disease with distinct biological and prognostic groupings, and the accurate classification is critical for treatment and prognosis. Classification of acute leukemia began with the observation of variability in clinical outcome and subtle differences in nuclear morphology. Diagnosis and classification have been based on cytomorphology and cytochemistry, which provide the basis for the classification of acute leukemia into those arising from lymphoid precursors [acute lymphoid leukemia $(A L L)]$ or from myeloid precursors [acute myeloid leukemia (AML)] [33]. To detect the methylation and expression of glioma pathogenesis-relatedprotein 1(GLIPR1) gene in the acute myeloid leukemia (AML) cell lines and bone marrow cells from AML patients, and to determine the relationship between promoter methylation and expression of GLIPR1 is downregulated or even lost by promoter methylation in AML, and the expression and methylation level of GLIPR1 gene may have some significance in evaluating the curative effect of AML patients [34]. It was concluded that the Irp16 gene is a leukemic oncogene and closely relates to genesis and progression of leukemia, which may be an incicator for evaluating clinical efficacy of leukemia therapy [35]. Proteomic analysis should be further explored to define factors important in predicting clinical response in patients with acute myeloid leukemia [36].

\section{Proteomics of prostate cancer}

Data derived from multiple proteomics platforms and transcript data coupled with informatics analysis provides a deeper insight into the functional consequences of androgen action in prostate cancer [37]. A proportion of men with prostate cancer will progress to develop metastatic disease involving the lymph-nodes and bone. To identify novel candidates associated with metastatic progression, researchers compared the proteomic profiles of LNCaP (lymph-node metastatic, androgendependant) and PC-3 in human prostate cancer cells [38]. Evidence supporting the hypothesis that prostate cancer arises from malignant transformation of intermediate stem cells included the presence in prostate cancers of keratin patterns associated with the intermediate stages of differentiation, androgen independence of both prostate cancers and intermediate stem cells, and expression of c-met by the TP/A intermediate stem cells and tumor cells [39]. Expression of $\mathrm{K} 5$ in the absence of K14 identifies the existence of an intermediate cell population in prostate carcinoma. Accumulation of intermediate cells in regressed and hormone-escaped prostate cancer indicates that for their survival, these cells are androgen-independent [40].

\section{Proteomics of ovarian and cervical cancer} Cervical cancer caused by the human papilloma virus (HPV). In 2D proteomic analysis, an increase of HSP60 expression was detected in cervical cancer tissues and confirmed by Western blot analysis $(p<0.05)$. However, messenger RNA (mRNA) levels of HSP60 did not display any significant differences between cervical cancer and normal tissues [41]. This study shows the power of proteomic profiling with functional analysis using RNAi technology for the discovery of novel molecular targets and a better understanding of the actions of paclitaxel at the molecular level in cervical carcinoma cells [42]. It was suggested that 5-FU suppresses the growth of cervical cancer cells not only by antiproliferative effect but also antiviral regulation. Recent findings may offer new insights into the mechanism of anticancer effect affected by 5-FU treatment in cervical cancer cells and its mode of action [43]. To date no biomarkers for early diagnosis or prognostication in ovarian cancer have been found using proteomics. We speculate that it would be interesting to investigate the tissue proteome in an attempt to overcome acute phase reactants and to facilitate the discovery of real tumor-specific biomarkers instead of the identification of secondary protein changes [45]. One major objective of the NCl-FDA Tissue Proteomics Initiative has been to combine the technique of laser capture microdissection (LCM) of epithelial tumor cells in human tissue specimens with two-dimensional gel electrophoresis (2-D PAGE) to identify proteins .LMP ovarian cancer may more directly generate important markers for early detection and therapeutic targets unique to the invasive 
phenotype [46]. As can be summerised proteomic profiling of differentially expressed proteins in the sera of normal women compared to women with ovarian cancer can greatly facilitate the discovery of a panel of biomarkers that may aid in the detection of ovarian cancer with greater specificity [47].

\section{References}

[1] Koomen J.M., Shih L.N., Coombes K.R., Li D., Xiao L.C., Fidler I.J., Abbruzzese J.L., Kobayashi R. (2005) Clin Cancer Res. 11(3), 1110-8.

[2] Goodison S., Rosser C.J., Urquidi V. (2009) Expert Rev Proteomics 6(5):507-514.

[3] Goodison S., Urquidi V.(2008) Expert Rev Proteomics, 5(3):457-67

[4] André X. C., Valente N., Roberts S.B., Buck G.A. and Yuan G. (2009) Proc Natl Acad Sci,106(5): 1490-1495.

[5] Ramadan E., Ward M., Guo X., Durkin S.S., Sawyer A., Vilela M., Osgood C., Pothen A., and Semmes O.J.(2008) Retrovirology, 5: 92

[6] Ammann L.P.,Goodman S.R.(2009) Experimental Biology and Medicine, 234(6):703 - 711.

[7] Fang X., Wallqvist A. and Reifman J. (2009) Systems Biology, 3:92doi:10.1186/17520509-3-92

[8] Ulitsky I., Shamir R. (2007) BMC Syst Biol. $26 ; 1: 8$

[9] Nikolsky Y., Kirillov E., Zuev R., Rakhmatulin E., Nikolskaya T.(2009) Methods Mol Biol 563: 177-96.

[10] Cakır T., Hendriks M.M., Westerhuis J.A., Smilde A.K.

(2009) Metabolomics.,5(3):318-329.

[11] Lubec G., Krapfenbauer K. and Fountoulakis M.(2003) Progress Neurobiol. 2003, 69, 193-211.

[12] Yang J.W., Suder P., Silberring J., Lubec G. (2005) Neurochem Int.,47(3):159-72.

[13] Weinberger P.M., Merkley M., Lee J.R., Adam B.L., Gourin C.G., Podolsky R.H., Haffty B.G., Papadavid E., Sasaki C., Psyrri A., Dynan W.S. (2009) Arch Otolaryngol Head Neck Surg.,135(7):694-703.

[14] Melle C., Ernst G., Schimmel B., Bleul A., Koscielny S., Wiesner A., Bogumil R., Moller U., Osterloh D., Halbhuber K.J., von Eggeling F. (2003) Mol Cell Proteomics,2(7):443-52.

[15] Wu W., Tang X., Hu W., Lotan R., Hong W.K., Mao L. (2002) Clin Exp Metastasis, 19(4):319-26.

[16] Zhu L., Ni G., Zhang Z., Xu X., Hu X., Li W. (2009) Se Pu,27(3):270-8.

[17] Zeng Z., Hincapie M., Haab B.B., Hanash S., Pitteri S.J., Kluck S., Hogan J.M.,
Kennedy J., Hancock W.S. (2009) J Chromatogr A. [PMID: 19782370].

[18] Antwi K., Hanavan P.D., Myers C.E., Ruiz Y.W., Thompson E.J., Lake D.F. (2009) Mol Immunol.,46(15):2931-7.

[19] Yasui Y., Tanaka T. (2009) J Carcinog., 8:10.

[20] Ma Y., Peng J., Liu W., Zhang P., Huang L., Gao B., Shen T., Zhou Y., Chen H., Chu Z., Zhang M., Qin H. (2009) Mol Cell Proteomics,8(8):1878-90.

[21] Krasnov G.S., Oparina Nlu, Khankin S.L., Mashkova T.D., Ershov A.N., Zatsepina O.G., Karpov V.L., Beresten' S.F. (2009) Mol Biol.,43(2):348-56.

[22] Brody J.S. and Avrum Spira State of the Art. (2006) The Proceedings of the American Thoracic Society 3:535-537

[23] Okano T., Kondo T., Kakisaka T., Fujii K., Yamada M., Kato H., Nishimura T., Gemma A, Kudoh S, Hirohashi S. (2006) Proteomics,6(13):3938-48.

[24] Nakajima Y., Nagai K., Miyake S., Ohashi K., Kawano T., Iwai T. (2002) J Cancer Res.,93(3):305-12.

[25] Uemura N., Nakanishi Y., Kato H., Saito S., Nagino M., Hirohashi S. (2009) Int J Cancer., 124(9):2106-15.

[26] Uemura N., Nakanishi Y., Kato H., Nagino M., Hirohashi S.; Kondo T. (2009) Cancer Science, 100(9),1612-1622(11).

[27] Sano A., Kato H., Sakurai S., Sakai M., Tanaka N., Inose T., Saito K., Sohda M., Nakajima M., Nakajima T., Kuwano H. (2009) Ann Surg Oncol. 16(2):506-14.

[28] Tang L., Yang J., Liu W., Tang X., Chen J., Zhao D., Wang M., Xu F., Lu Y., Liu B., Sun Q., Zhang L., He F. (2009) Gastroenterology,137(4):1498-508.

[29] Luo X.H., Yang Q., Zhang Q., Cheng M.L. (2007) Zhonghua Yi Xue Za Zhi. 25;87(48):3411-4.

[30] Patwa T.H., Li C., Poisson L.M., Kim H.Y., Pal M., Ghosh D., Simeone D.M., Lubman D.M. (2009) Electrophoresis, 30(12):2215-26.

[31] Fiedler G.M., Leichtle A.B., Kase J., Baumann S., Ceglarek U., Felix K., Conrad T., Witzigmann H., Weimann A., Schütte C., Hauss J., Büchler M., Thiery J. (2009) Clin Cancer Res.,15(11):38129.

[32] Hong S.H., Misek D.E., Wang H., Puravs E., Giordano T.J., Greenson J.K., Brenner D.E., Simeone D.M., Logsdon C.D., Hanash S.M.(2004) Cancer Res.,64(15):5504-10.

[33] Jiu-wei cui, Jie wang, kun He. (2004) Clinical cancer research , 10, 68876896.

[34] Liang T., Tan T., Xiao Y., Yi H., Li C., Peng F., Chen Z., Xiao Z. (2009) Zhong Nan 
Da Xue Xue Bao Yi Xue Ban., 34(5):388-94.

[35] Yang B., Chi X.H., Lu X.C., Han W.D., Yu L., Lou F.D. (2009) Zhongguo Shi Yan Xue Ye Xue Za Zhi.,17(4):857-60.

[36] Albitar M., Potts S.J., Giles F.J., O'Brien S., Jilani I., Donahue A.C., Estey E.H.,Kantarjian H. (2009) Exp Hematol.,37(7):784-90.

[37] Vellaichamy A., Sreekumar A., Strahler J.R., Rajendiran T., Yu J., Varambally S., Li Y., Omenn G.S., Chinnaiyan A.M., Nesvizhskii A.I. (2009) PLoS One, 18;4(9):e7075.

[38] Leiblich A., Cross S.S., Catto J.W., Pesce G., Hamdy F.C., Rehman I. (2007) Prostate.,67(16):1761-9.

[39] Schalken J.A., Van Leenders G. (2003) Urology,62:11-20.

[40] Van Leenders G.J., Aalders T.W., Hulsbergen-van de Kaa C.A., Ruiter D.J., Schalken J.A. (2001) J Pathol., 195(5):563-70.

[41] Hwang Y.J., Lee S.P., Kim S.Y., Choi Y.H., Kim M.J., Lee C.H., Lee J.Y., Kim D.Y.(2009) Yonsei Med J. 30;50(3):399406.

[42] Lee K.H., Yim E.K., Kim C.J., Namkoong S.E., Um S.J., Park J.S. (2005) Gynecol Oncol.,98(1):45-53.

[43] Yim E.K., Lee K.H., Bae J.S., Namkoong S.E., Um S.J., Park J.S. (2004) DNA Cell Biol.,23(11):769-76.

[44] Cadron I., Van Gorp T., Timmerman D., Amant F., Waelkens E., Vergote I. (2009) Gynecol Oncol., 115(3):497-503.

[45] Jones M.B., Krutzsch H., Shu H., Zhao Y., Liotta L.A., Kohn E.C. Petricoin E.F. 3rd. (2002) Proteomics, 2(1):76-84.

[46] Ahmed N., Oliva K.T., Barker G., Hoffmann P., Reeve S., Smith I.A., Quinn M.A., Rice G.E. (2005) Proteomics, 5(17):4625-36.

[47] Purcell A., Fleming J., Bennett S., McGuane K., Burmeister B., Haines T.A. (2009) Cancer, [PMID: 19921735]

[48] Braun K., Wiessler M., Ehemann V., Pipkorn R., Spring H., Debus J., Didinger B., Koch M., Muller G., Waldeck W. (2009) Drug Des De vel Ther., 2:289-301.

[49] Chen C., Jin J., James D.A., Adams-Cioaba M.A., Park J.G., Guo Y., Tenaglia E., Xu C., Gish G., Min J., Pawson T. (2009) Proc Natl Acad Sci. [PMID: 19918066]

[50] Neumann K., Oellerich T., Urlaub H., Wienands J. (2009) Immunol Rev., 232(1):135-49.

[51] van Lunteren E., Moyer M. (2009) PLoS One, 4(11):e7832.
[52] Krijgsveld J., Tops B.B., Gauci S., Heck A.J.(2009) J Proteome Res., [PMID: 19916504].

[53] Caspi R., Altman T., Dale J.M., Dreher K., Fulcher C.A., Gilham F., Kaipa P., Karthikeyan A.S., Kothari A., Krummenacker M., Latendresse M., Mueller L.A., Paley S., Popescu L., Pujar A., Shearer A.G., Zhang P., Karp P.D. (2009) Nucleic Acids Res., [PMID: 19850718].

[54] Zaparty M., Esser D., Gertig S., Haferkamp P., Kouril T., Manica A., Pham T.K., Reimann J., Schreiber K., Sierocinski P., Teichmann D., van Wolferen M., von Jan M., Wieloch P., Albers S.V., Driessen A.J., Klenk H.P., Schleper C., Schomburg D., van der Oost J., Wright P.C., Siebers B. (2009) Extremophiles., [PMID: 19802714].

[55] Sakata K., Ohyanagi H., Nobori H., Nakamura T., Hashiguchi A., Nanjo Y., Mikami Y., Yunokawa H., Komatsu S. (2009) J Proteome Res.,8(7):3539-48.

[56] McGarvey P.B., Huang H., Mazumder R., Zhang J., Chen Y., Zhang C., Cammer S., Will R., Odle M., Sobral B., Moore M., Wu C.H. (2009) PLoS One, 25;4(9):e7162.

[57] Bulanova E., Bulfone-Paus S.(2009) Purinergic Signal.,[ PMID: 19921464]. 